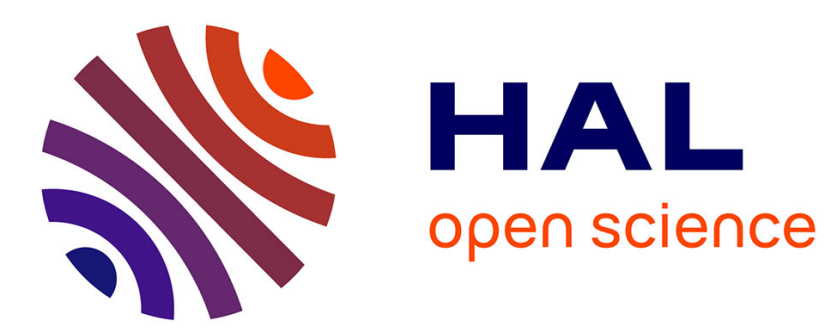

\title{
Evolution of Image Regularization with PDEs toward a New Anisotropic Smoothing based on Half Kernels
}

\author{
Baptiste Magnier, Philippe Montesinos
}

\section{To cite this version:}

Baptiste Magnier, Philippe Montesinos. Evolution of Image Regularization with PDEs toward a New Anisotropic Smoothing based on Half Kernels. IS\&T/SPIE Electronic Imaging 2013, Feb 2013, San Francisco, United States. http://spie.org/. hal-00807826

\section{HAL Id: hal-00807826 \\ https://hal.science/hal-00807826}

Submitted on 4 Apr 2013

HAL is a multi-disciplinary open access archive for the deposit and dissemination of scientific research documents, whether they are published or not. The documents may come from teaching and research institutions in France or abroad, or from public or private research centers.
L'archive ouverte pluridisciplinaire HAL, est destinée au dépôt et à la diffusion de documents scientifiques de niveau recherche, publiés ou non, émanant des établissements d'enseignement et de recherche français ou étrangers, des laboratoires publics ou privés. 


\title{
Evolution of Image Regularization with PDEs toward a New Anisotropic Smoothing based on Half Kernels
}

\author{
Baptiste Magnier $^{a}$ and Philippe Montesinos ${ }^{a}$ \\ ${ }^{a}$ LGi2P de l'Ecole des Mines d'Alès, Parc scientifique G. Besse, 30035 Nîmes cedex 1
}

\begin{abstract}
This paper is dedicated to a new anisotropic diffusion approach for image regularization based on a gradient and two diffusion directions obtained from half Gaussian kernels. This approach results in smoothing an image while preserving edges. From an anisotropic edge detector, built of half Gaussian derivative kernels, we introduce a new smoothing method preserving structures which drives the diffusion function of the angle between the two edge directions and the gradient value. Due to the two directions diffusion used in the control function, our diffusion scheme enables to preserve edges and corners, contrary to other anisotropic diffusion methods. Moreover, parameters of the Gaussian kernel can be tuned to be sufficiently thin extracting precisely edges whereas its length allows detecting in contour orientations which leads to a coherent image regularization. Finally, we present some experimental results and discuss about the choice of the different parameters.
\end{abstract}

Keywords: Anisotropic smoothing, diffusion PDEs, half Gaussian kernel, anisotropic edge detector

\section{PARTIAL DIFFERENTIAL EQUATIONS AND ANISOTROPIC DIFFUSION}

Image regularization of noisy, corrupted or degraded images caused for example by compression artifacts is a field that has largely benefited from techniques of Partial Differential Equations (PDE) ${ }^{1,2}$. PDEs belong to one of the most important part of mathematical analysis and are closely related to the physical world. In this context, images are considered as evolving functions of time and a regularized image can be seen as a version of the original image at a special scale. In this paper, let us note $I: \Omega \rightarrow \mathbb{R},\left(\Omega \subset \mathbb{R}^{2}\right)$ a grey level image with $I(x, y)$ corresponding to the pixel intensity of coordinates $(x, y)$. The general evolution model can be formally written in the following form:

$$
\left\{\begin{array}{l}
\frac{\partial I}{\partial t}(x, y, t)=F\left(I(x, y, t), \frac{\partial I}{\partial x}(x, y, t), \frac{\partial I}{\partial y}(x, y, t), \frac{\partial^{2} I}{\partial x^{2}}(x, y, t), \frac{\partial^{2} I}{\partial y 2}(x, y, t), \frac{\partial^{2} I}{\partial x \partial y}(x, y, t)\right) \\
I(x, y, 0)=I_{0}(x, y)
\end{array}\right.
$$

where $I_{0}$ represents the original image and $F$ is a control function of the diffusion, penalizing high gradients, while preserving edges, depending of $I_{0}$ and its spatial derivatives of the first or second order ${ }^{3}$.

It should be noted that Koenderink ${ }^{4}$ was the first to underline the equivalence between the convolution with a Gaussian kernel of standard deviation $\sqrt{2 t}$ and the solution of the PDE describing the heat diffusion ${ }^{4}$, at a time $t$ :

$$
\begin{cases}\frac{\partial I}{\partial t}(x, y, t) & =\Delta I=\frac{\partial^{2} I}{\partial x^{2}}+\frac{\partial^{2} I}{\partial y^{2}} \\ I(x, y, 0) & =I_{0}(x, y)\end{cases}
$$

This smoothing process, called isotropic diffusion, is known to smooth noise and blur edges, leading to loose image structures. In order to regularize images by controlling the diffusion, Perona and Malik ${ }^{5}$ have proposed a model described by the following equation:

$$
\begin{cases}\frac{\partial I}{\partial t}(x, y, t) & =\operatorname{div}(g(\|\nabla I\|) \cdot\|\nabla I\|) \\ I(x, y, 0) & =I_{0}(x, y)\end{cases}
$$

Further author information:

E-mail: \{baptiste.magnier, philippe.montesinos\}@mines-ales.fr 
where div represents the divergence* operator and $g(s):[0,+\infty[\rightarrow] 0,+\infty[$ a decreasing function satisfying $g(0)=1$ and $g(+\infty)=0$, this function could be chosen as:

$$
g(\|\nabla I\|)=e^{\left(-\frac{\|\nabla I\|}{K}\right)^{2}}
$$

with $K \in \mathbb{R}$ a constant that can be assimilated to a gradient threshold or a diffusion barrier.

The decomposition of the eq. 3 with the second derivatives of $I$ in orthogonal directions $(\xi \perp \eta)$ respectively in the edge direction called $\xi$ and in the gradient direction labelled $\eta=\frac{\nabla I}{\|\nabla I\|}$ enables to understand the diffusion behavior ${ }^{6}$ :

$$
\left\{\begin{array}{l}
\frac{\partial I}{\partial t}(x, y, t)=c_{\xi} \cdot I_{\xi \xi}+c_{\eta} \cdot I_{\eta \eta} \\
I(x, y, 0)=I_{0}(x, y)
\end{array}\right.
$$

where $\left(I_{\xi \xi}, I_{\eta \eta}\right)=\left(\frac{\partial^{2} I}{\partial \xi^{2}}, \frac{\partial^{2} I}{\partial \eta^{2}}\right), c_{\xi}$ and $c_{\eta}$ are coefficients tuning the diffusion (diagrammed in Fig. 1(a)). When $c_{\xi \xi}=c_{\eta \eta}=1$, the eq. 6 is equivalent to the heat equation (i.e. eq. 2). Choosing $c_{\xi}=g(\|\nabla I\|)$, a gradient function and $c_{\eta}=g(\|\nabla I\|)+\|\nabla I\| \cdot g^{\prime}(\|\nabla I\|)$, the diffusion process described in eq. 6 can be interpreted as two directional heat flows ${ }^{\dagger}$ with different diffusion intensities in the $\eta$ and $\xi$ directions to preserve discontinuities:

- Inside homogeneous regions, the gradient magnitude $\|\nabla I\|$ is small and the diffusion is isotropic.

- On edges, the diffusion becomes anisotropic, being attenuated by the function $g$, and is inhibited when coefficients $\left(c_{\xi \xi}, c_{\eta \eta}\right)$ tend to zero.

Diffusion control is done with finite differences so that many contours of small objects or small structures are preserved. However, with highly noisy images, generally, the noise is not totally removed because the diffusion process is inhibited and it may generate a lot of undesired artifacts.

In order to make the Perona-Malik filter less sensitive to noise and more stable, a number of works were elaborated using Gaussian filtering for gradient estimation. Several authors took the approach of eq. 6 for imposing specific actions along the two diffusion axes using Gaussian filter. We can mention here the approach of Alvarez et al. ${ }^{7}$ which induces for each pixel an adaptive unidirectional diffusion at level of edges or efficient isotropic smoothing for noise removal inside homogeneous regions using the following PDE:

$$
\left\{\begin{array}{l}
\frac{\partial I}{\partial t}(x, y, t)=g\left(\left\|\nabla I_{\sigma}\right\|\right) \cdot\left[I_{\xi \xi}+(1-h(\|\nabla I\|)) \cdot I_{\eta \eta}\right] \\
I(x, y, 0)=I_{0}(x, y)
\end{array}\right.
$$

with $I_{\sigma}$ denoting a smoothed version of the original image $I_{0}$ using a Gaussian filter of standard deviation $\sigma$. The $g$ function could be the same as in the eq. 5 , controlling the diffusion rate. Although this approach brings interesting results for images presenting a low noise, concerning high level of noise, this diffusion method either smooths isotropically structures having a small gradient in the image, or preserves some noisy pixels having a strong gradient. Moreover, the $h$ function does not allow a progressive diffusion in the gradient direction $\eta$ :

$$
h(\|\nabla I\|)= \begin{cases}0 & \text { if } \quad\|\nabla I\| \leqslant e \\ (\|\nabla I\|-e) / e & \text { if } e<\|\nabla I\|<2 e \\ 1 & \text { if } 2 e \leqslant\|\nabla I\|\end{cases}
$$

${ }^{*}$ Let be $f$ a function such that $f: \Omega \subset \mathbb{R}^{2} \rightarrow \mathbb{R}^{2}$, then $\operatorname{div}(f)=\frac{\partial f}{\partial x}+\frac{\partial f}{\partial y}$.

Note that the heat equation can also be written as a divergence:

$$
\begin{cases}\frac{\partial I}{\partial t}(x, y, t) & =\operatorname{div}(\nabla I) \\ I(x, y, 0) & =I_{0}(x, y)\end{cases}
$$

${ }^{\dagger}$ Note that if $\|\nabla I\|>K / \sqrt{2}$, then $c_{\eta}<0$ and the anisotropic diffusion equation behaves locally like an inverse diffusion equation which is an unstable process enhancing features. 
with $e \in \mathbb{R}$ a positive constant generally small (close to zero). For gradient value less than $e$ the diffusion is isotropic ; if the gradient is greater than $2 e$, the diffusion only depends on the tangential derivative $I_{\xi \xi}$. Consequently, in the presence of a high noise, even in homogeneous regions, this diffusion scheme behaves like the Mean Curvature Motion ${ }^{8}(\mathrm{MCM})$ method which consists in performing the diffusion only along the tangential direction $\xi$ or along isophotes (i.e. curves of the image surface of constant intensity):

$$
\left\{\begin{array}{lll}
\frac{\partial I}{\partial t}(x, y, t) & =c_{\xi} \cdot I_{\xi \xi} \\
I(x, y, 0) & =I_{0}(x, y)
\end{array}\right.
$$

with $c_{\xi}=1$ for the $M C M$ scheme and $c_{\xi}=g\left(\left\|\nabla I_{\sigma}\right\|\right)$ in Alvarez et al. ${ }^{7}$. Although the $M C M$ scheme regularizes the image in edge directions, this approach tends to round corners after a certain number of iterations.

Instead of considering only the gradient magnitude to tune the diffusion, tensorial approaches ${ }^{9-11}$ contribute to another image diffusion formalism. This formulation relies on the definition of a tensor field that imposes the smoothing directions. From a structure tensor $J_{\rho}=G_{\rho} * \nabla I_{\sigma} \nabla I_{\sigma}^{T}$, where $G_{\rho}$ denotes a Gaussian kernel of standard deviation $\rho$, authors of ${ }^{9-11}$ elaborate a tensor field $\mathbf{T}$ which specifies the local smoothing geometry defined from its spectral elements. Then, using the divergence ${ }^{9}$ or the $\operatorname{trace}^{10}$, the smoothing process is realized using the following PDEs:

$$
\left\{\begin{array} { l } 
{ \frac { \partial I } { \partial t } ( x , y , t ) = \operatorname { d i v } ( \mathbf { T } \nabla I ) } \\
{ I ( x , y , 0 ) = I _ { 0 } ( x , y ) }
\end{array} \quad \text { or } \quad \left\{\begin{array}{l}
\frac{\partial I}{\partial t}(x, y, t)=\operatorname{trace}(\mathbf{T H}) \\
I(x, y, 0)=I_{0}(x, y)
\end{array}\right.\right.
$$

where $\mathbf{H}$ represents the Hessian matrix of $I$. Then, the smoothing along a contour in inversely proportional to the contour strength in the direction of the eigenvector associated to the higher eigenvalue. Inside homogeneous regions, eigenvalues are close to zero and the diffusion becomes isotropic.

As demonstrated ${ }^{10}$, trace based PDE is best suited to understand the local smoothing geometry behavior. These tensorial diffusion schemes ensure coherence smoothing directions but the Gaussian behavior on curved structures or corners results in a "mean curvature flow effect" leading to round small structures or corners. In order to compensate this drawback, the author of ${ }^{11}$ proposed a curvature-preserving smoothing PDE that diffuses $I$ along a field of vectors $\mathbf{w}$ issued of the eigenvectors of $J_{\rho}$ :

$$
\left\{\begin{array}{l}
\frac{\partial I}{\partial t}(x, y, t)=\operatorname{trace}\left(\mathbf{w w}^{\mathbf{T}} \mathbf{H}\right)+\nabla I^{T} J_{\mathbf{w}} \mathbf{w} \\
I(x, y, 0)=I_{0}(x, y)
\end{array}\right.
$$

where $J_{\mathbf{w}}$ is the Jacobian matrix of $\mathbf{w}$. Despite the fact that the author of this method ${ }^{11}$ has demonstrated that it better preserves corners and small structures in the image, as the other tensorial approaches, when the anisotropic coefficient is too large, the diffusion of a high noise brings a fiber effect in homogeneous regions. To

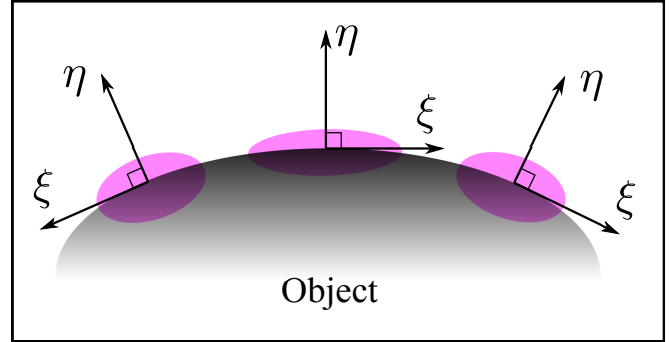

(a) Gradient and tangential direction denoted $(\eta, \xi)$

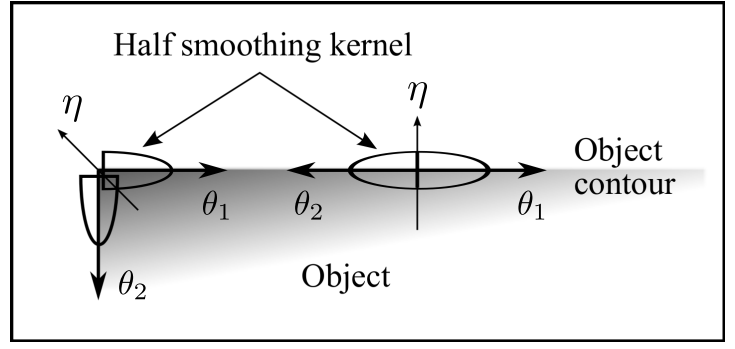

(b) Half anisotropic gaussian kernel

Figure 1. Diagrams of edge directions. (a) An image contour and its moving vector basis $(\xi, \eta)$ and diffusion representation with ellipsoids. The more the gradient is high, the more the ellipse is ellongated. Note that ellipsoids are not always oriented in the $\xi$ direction using tensorial methods. (b) Directions of the edges using half anisotropic gaussian kernels. 
avoid this undesired diffusion effect, it is preferable to use a higher standard deviation of the Gaussian $\sigma$, however this leads to delocate even so the corners and blur edges.

In this paper, we propose a new PDE scheme that regularizes images considering two contour directions. This diffusion process correctly preserves corners as well as small objects and removes noise inside homogeneous regions without generating undesired fiber effect. Thanks to a rotating Gaussian derivative half-filter, we extract a gradient amplitude and determine two edge directions. Then we apply an anisotropic diffusion on each pixel using new control functions adapted to our objectives of image regularization.

\section{A GRADIENT EXTRACTION AND TWO EDGE DIRECTIONS ESTIMATION}

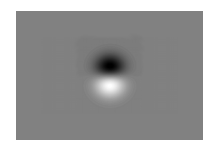

(a) Isotropic gaussian kernel

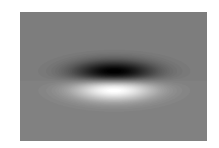

(b) Anisotropic gaussian kernel

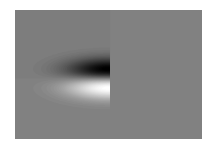

(c) Half anisotropic gaussian kernel

Figure 2. Different 2D derivative Gaussian kernels

Steerable filters ${ }^{12,13}$ or anisotropic edge detectors ${ }^{14}$ perform well in detecting large linear structures. Close to corners however, the gradient magnitude decreases as the edge information under the scope of the filter decreases. Consequently, the robustness to noise concerning small objects becomes inappropriate.

A simple solution to bypass this effect is to consider paths crossing each pixel in several directions as in ${ }^{15}$. Wedge steerable filters introduced by Simoncelli and Farid ${ }^{16}$ are composed of asymmetric masks providing orientation of edges in different directions issued from a pixel. Unlike the Gaussian function, which is an optimal solution for the Canny criteria ${ }^{17}$, wedge steerable filters have a constant amplitude on almost the whole extent of the mask. The idea developed in ${ }^{18}$ was to split the derivative (and smoothing) anisotropic Gaussian kernel in two parts: a first part along an initial direction, and a second part along a second direction (represented in Fig. 1 (a)). As diagrammed in Fig. 3(a), at each pixel of coordinates $(x, y)$, a derivation filter is applied to obtain a derivative information $\mathcal{Q}(x, y, \theta)$ in function of the orientation $\theta \in[0 ; 2 \pi[$ :

$$
\mathcal{Q}(x, y, \theta)=I_{\theta} * C \cdot H(-y) \cdot x \cdot e^{-\left(\frac{x^{2}}{2 \lambda^{2}}+\frac{y^{2}}{2 \mu^{2}}\right)}
$$

where $I_{\theta}$ corresponds to a rotated image ${ }^{\ddagger}$ of orientation $\theta, C$ is a normalization coefficient, $(x, y)$ are pixel coordinates, and $(\mu, \lambda)$ the standard deviations of the anisotropic Gaussian filter. Since we only require the

\footnotetext{
${ }^{\ddagger}$ As explained $\mathrm{in}^{18}$, the image is oriented instead of the filter so as to increase algorithmic complexity and moreover allows use of a recursive Gaussian filter ${ }^{19}$.
}

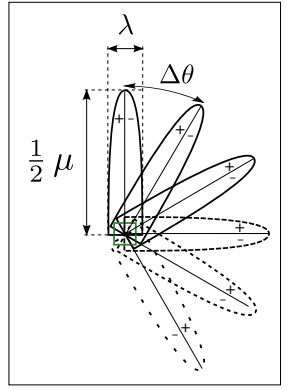

(a) Rotated derivation filter

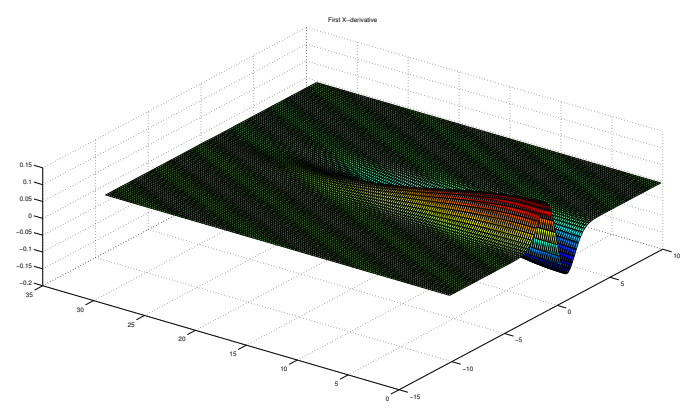

(b) Half Gaussian derivative representation

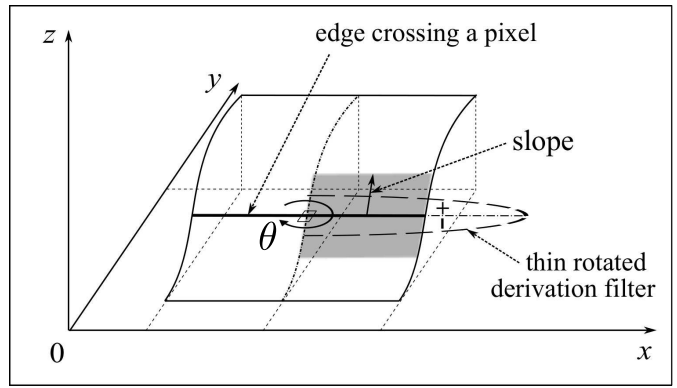

(c) Estimation of the slope turning around a pixel.

Figure 3. A thin rotating Gaussian derivative half-filter. For (b): $\mu=10$ and $\lambda=1$. For (c): the $z$ axis represents the pixel intensity. 


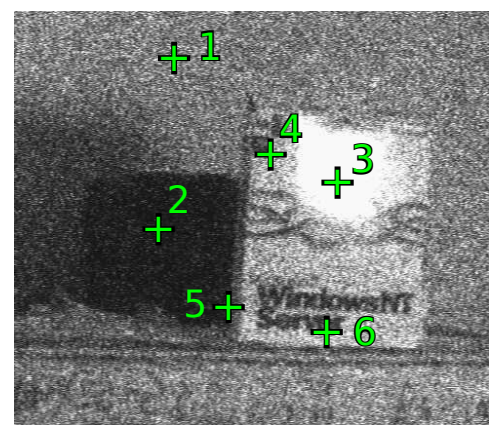

(a) Points selection
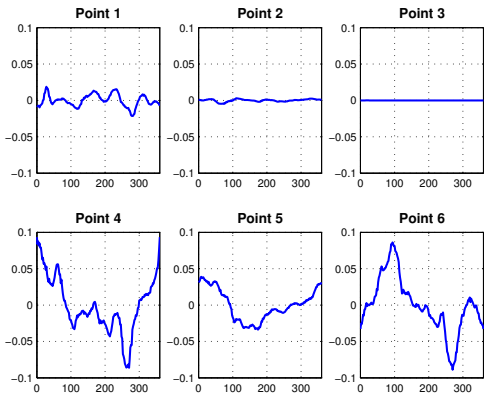

(b) $\mathcal{Q}(x, y, \theta)$ for each points of (a)

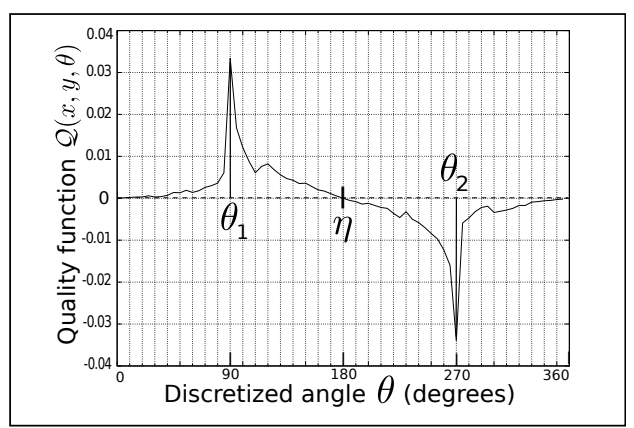

(c) Extrema of a function $\mathcal{Q}(x, y, \theta)$

Figure 4. Points selection and its associated $\mathcal{Q}(x, y, \theta), \mu=10, \lambda=1$ and $\Delta \theta=2^{\circ}$. Note that the initial orientation of the filter is vertical, upwardly directed and steerable clockwise.

causal part of this filter along $Y$ axis, we simply "cut" the smoothing kernel by the middle, in an operation that corresponds to the Heaviside function $H$.

This filter is represented in Fig. 3(b) and can be compared with isotropic and full anisotropic derivative Gaussian kernels in Fig. 2. As diagrammed in Fig. 3(b), $\mathcal{Q}(x, y, \theta)$ represents the slope of a line derived from a pixel in the perpendicular direction to $\theta$ (see Fig. 4(b) for several $\mathcal{Q}(x, y, \theta)$ signals). We can note that similar filters can also be used for the matching of interest points ${ }^{20}$.

To obtain a gradient $\|\nabla I\|$ and its associated direction $\eta$ on each pixel $P$, we first compute global extrema of the function $\mathcal{Q}(x, y, \theta)$, with $\theta_{1}$ and $\theta_{2} . \theta_{1}$ and $\theta_{2}$ define a curve crossing the pixel (an incoming and outgoing direction). Two of these global extrema can then be combined to maximize $\|\nabla I\|$, i.e. :

$$
\left\{\begin{aligned}
\|\nabla I\| & =\max _{\theta \in[0,2 \pi[} \mathcal{Q}(x, y, \theta)-\min _{\theta \in[0,2 \pi[} \mathcal{Q}(x, y, \theta) \\
\theta_{1} & =\underset{\theta \in[0,2 \pi[}{\arg \max }(\mathcal{Q}(x, y, \theta)) \\
\theta_{2} & =\underset{\theta \in[0,2 \pi[}{\arg \min }(\mathcal{Q}(x, y, \theta))
\end{aligned}\right.
$$

Fig. 5 shows two gradient images obtained using half Gaussian kernels. Once $\|\nabla I\|, \theta_{1}$ and $\theta_{2}$ have been obtained, the edges can be easily extracted by computing local maxima of $\|\nabla I\|$ in the direction of the angle $\eta$ (Fig. 4(c) and 6(a)) corresponding to the angle bisector between the two directions $\left(\theta_{1}, \theta_{2}\right)$ :

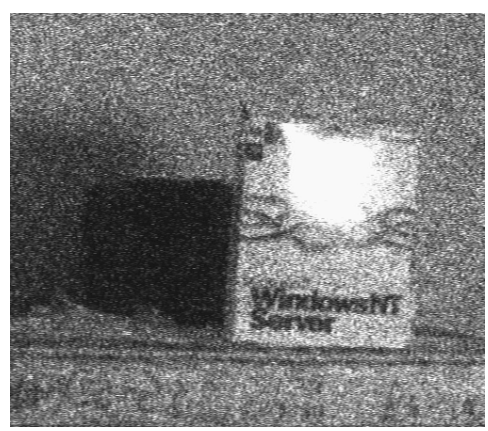

(a) Real noisy image containing high noise $508 \times 440$

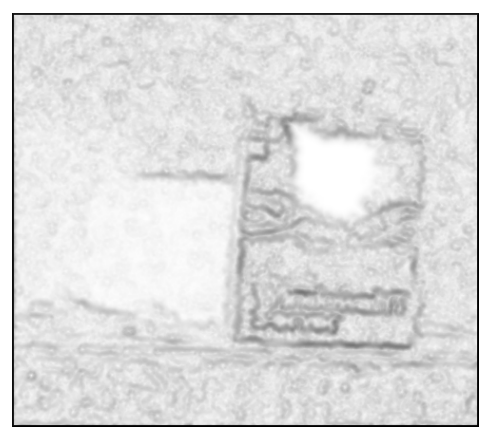

(b) Gradient image $\mu=5, \lambda=2, \Delta \theta=\frac{\pi}{90}$ (negative image)

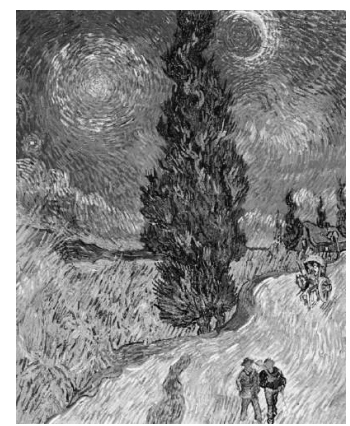

(c) "Road with Cypress and Star" by van Gogh $400 \times 503$

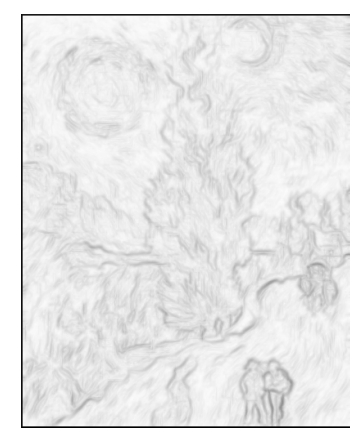

(d) Gradient image $\mu=5, \lambda=1, \Delta \theta=\frac{\pi}{90}$ (negative image)

Figure 5. Gradient images. 


$$
\eta=\frac{\theta_{1}+\theta_{2}}{2}
$$

Then, a binary image is built using an hysteresis threshold ( $\mathrm{see}^{18}$ for further details). In this paper, we are solely interested in the three directions $\left(\theta_{1}, \theta_{2}, \eta\right)$ and the gradient magnitude (see diagrams in Figs. 1(b) and $6(\mathrm{a}))$ used in our diffusion scheme discussed below.

Finally, due to their thinness, rotating filters enable computing two precise diffusion orientations in the edge directions, even at high noise levels ${ }^{21}$. $\mathrm{In}^{22}$, the authors have evaluated the edge detection used in this method with a strong noise level and a comparison with other approaches ${ }^{14,19}$ shows the efficiency of this method.

\section{ANISOTROPIC DIFFUSION IN TWO DIRECTIONS OF EDGES}

\subsection{A First Approach Preserving Edges and Smoothing Regions}

PDE-based image regularization techniques using gradient intensities of tensorial diffusion smooth the image either both in the directions of edges $\xi$ and gradient direction $\eta$ or in the directions provided by the eigenvectors of the tensor. However, all these approaches do not take account of the two directions of edges, for example at a level of a corner.

The original idea developed $\mathrm{in}^{23}$ for removing texture preserving edges was to smooth the image in the two diffusion directions $\left(\xi_{1}, \xi_{2}\right)$ issued of an edge classifier only at level of edges, and to smooth isotropically elsewhere:

$$
\left\{\begin{array}{l}
\frac{\partial I}{\partial t}(x, y, t)=I_{\xi_{1} \xi_{2}} \\
I(x, y, 0)=I_{0}(x, y)
\end{array}\right.
$$

where $I_{\xi_{1} \xi_{2}}=\frac{\partial^{2} I}{\partial \xi_{1} \partial \xi_{2}}$. This approach, efficient concerning the suppression of texture is not adapted for image regularization, especially because the diffusion is not controlled. In, ${ }^{21}$ after a pixel classification which determines roughly if a pixel belongs to a homogenous region or an edge, authors have developed a new diffusion method. Inside edge regions, a function of the gradient magnitude (eq. 13) and also of the angle between the two diffusion directions $\left(\theta_{1}, \theta_{2}\right)$ called $\beta$ drives the diffusion process:

$$
\begin{cases}\frac{\partial I}{\partial t}(x, y, t) & =f_{c} \cdot I_{\theta_{1} \theta_{2}} \\ f_{c} & \left.\left.=\frac{e^{-\left(\frac{\|\nabla I\|}{K_{1}}\right)^{2}}+e^{-\left(\frac{(\pi-\beta)}{\left(\pi \cdot K_{2}\right)}\right)^{2}}}{2} \text { with } K_{i, i \in\{1,2\}} \in\right] 0 ; 1\right] \\ I(x, y, 0) & =I_{0}(x, y)\end{cases}
$$

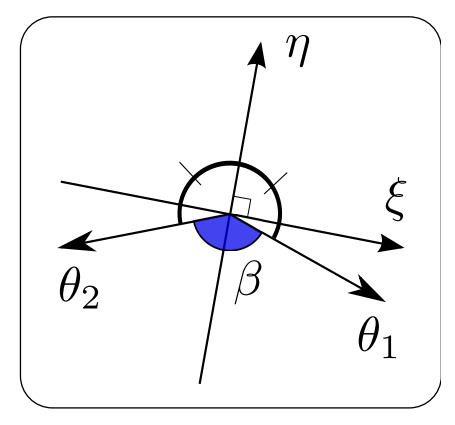

(a) Different directions and angles

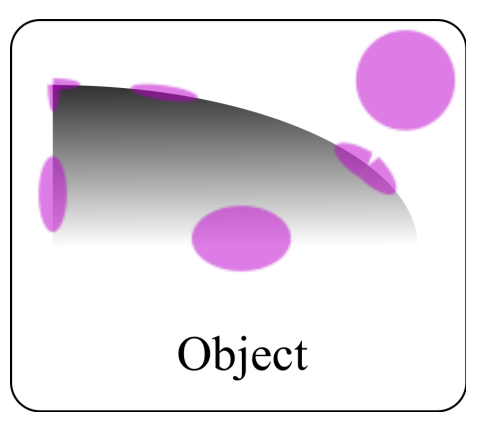

(b) Diffusion representation with half ellipsoids

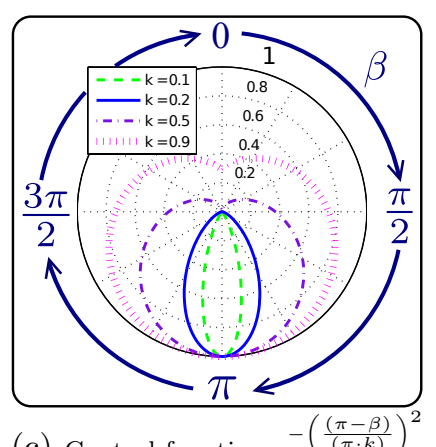

(c) Control function $e^{-\left(\frac{(\pi-\beta)}{(\pi \cdot k)}\right)^{2}}$

Figure 6. Different angles representation, our anisotropic diffusion scheme and polar representation of the angle control function. (b) The more the edge is sharped and the angle is acute, the more the half ellipses are thin. 
where $I_{\theta_{1} \theta_{2}}=\frac{\partial^{2} I}{\partial \theta_{1} \partial \theta_{2}}$. As diagrammed in Fig. 6(a), $\beta$ represents the angle between $\theta_{1}$ and $\theta_{2}$ such that $\beta=\operatorname{abs}\left(\theta_{1}-\theta_{2}\right)$ and its associated control function is represented in Fig. 6(c). This diffusion scheme presented in eq. 16 is efficient only at position of edges because in homogeneous noisy regions, it creates undesired lineaments caused by the directional diffusion $\left(\theta_{1}, \theta_{2}\right)$. Indeed, to fill this gap, the authors of ${ }^{21}$ use an isotropic diffusion in regions where edges are not detected (see eq. 2 ).

The diffusion process of ${ }^{21}$ accurately combines isotropic and anisotropic diffusion, while maintaining the edges and corners of different objects in highly noisy images. Nevertheless, instead of regularize textures, the use of the heat equation outside of rough edges regions smooths them isotropically. Moreover, as the anisotropic diffusion process is applied only at position of edges, it creates undesired artifacts near edges.

\subsection{A Diffusion Equation Driven by the Gradient Magnitude and Two Edge Directions}

In our method, we aim to remove noise or irregular artifacts in the original image $I_{0}$. Our algorithm enables a smoothing in the contour directions preserving edges $\left(I_{\theta_{1} \theta_{2}}\right.$ term) while diffusing also in the direction of $\eta$ for edges having a low gradient or inside homogeneous regions $\left(I_{\eta \eta}\right.$ term). Furthermore, these three directions smoothing terms have to be controlled in order to preserve image contours and not to create undesired artifacts or fiber effect elsewhere. In this respect, we propose a new PDE involving the gradient value and the $\beta$ angle driving both the diffusion terms $I_{\theta_{1} \theta_{2}}$ and $I_{\eta \eta}$ :

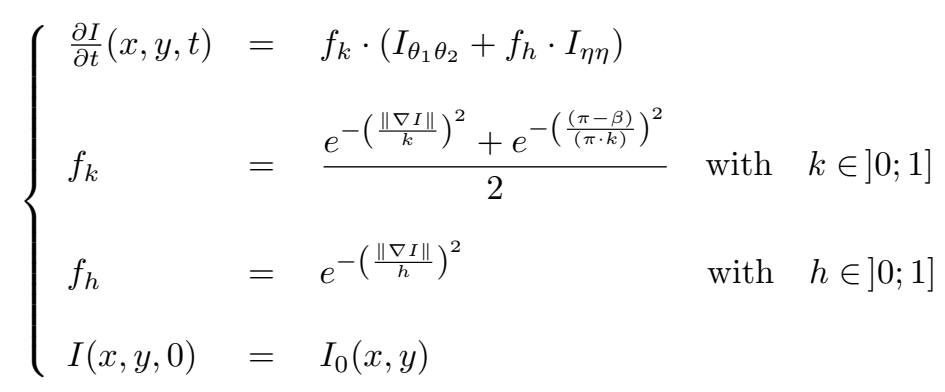

The smoothing process is driven by the gradient magnitude (eq. 13) and $\beta=\operatorname{abs}\left(\theta_{1}-\theta_{2}\right.$ ). The $f_{k}$ function ensure the diffusion preserving edges and corners whereas the $f_{h}$ function (same function that $g$ in eq. 5 ) enables a permanent smoothing in the gradient direction for noisy homogeneous regions. Contrary to the $h$ function ${ }^{7}$ (eq. 8), these control functions are not threshold functions but continuous functions (they are represented in Fig. 7 and Fig. 6(c)). Thus, the diffusion is never only in the $\left(\theta_{1}, \theta_{2}\right)$ directions, unlike the diffusion scheme of Magnier et al. ${ }^{21}$ (eq. 16). In case of a small gradient and a $\beta$ angle close to $\pi$, the considered pixel will be largely diffused (see Fig. 7(a)). If the gradient is important and the $\beta$ angle is small, smoothing is small and operates mainly along two directions $\left(\theta_{1}, \theta_{2}\right)$, as shown in Fig. 6(b).

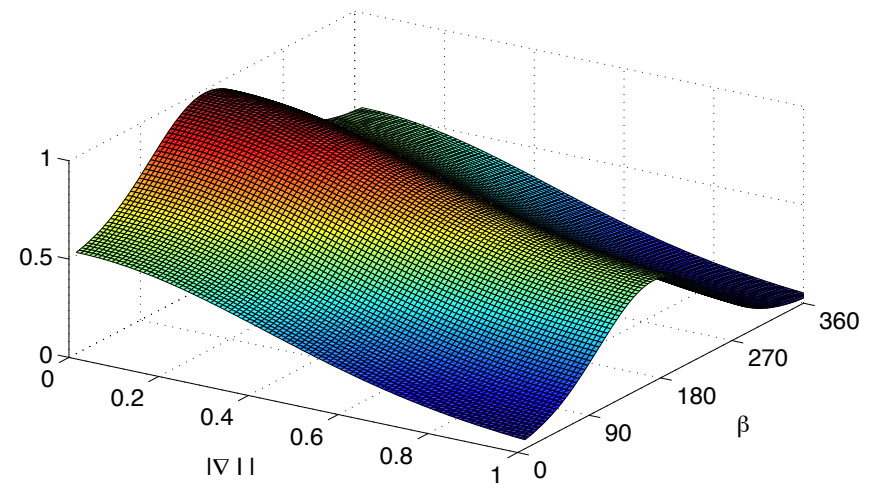

(a) $f_{k}$ function with $k=0.6$

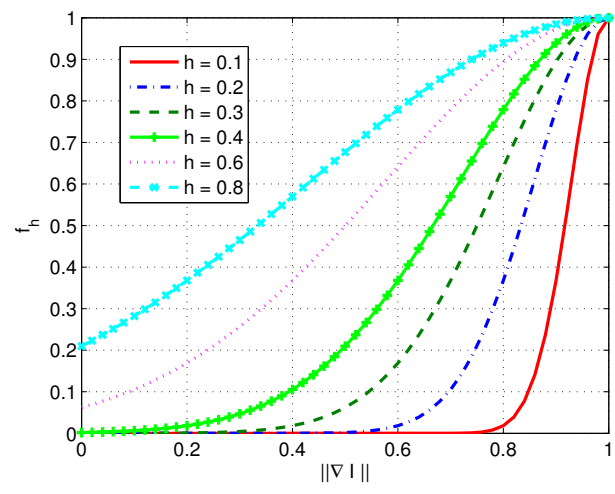

(b) $f_{h}$ function with different values of $h$

Figure 7. Control functions $\left(f_{k}, f_{h}\right)$. 


\section{EXPERIMENTAL RESULTS AND ANALYSIS}

In this section, we present several application results of our regularization method compared to different other approaches described above. For each result, presented below, we detail the parameters used either for our algorithm, or for other methods. Also, we show SSIM ${ }^{24}$ and PSNR representation of noisy images as a function of the number of iterations using different parameters which permit us to discuss about the choice of the best parameters couple $(k, h)$. Note that, in order to obtain precise diffusion directions $\left(\theta_{1}, \theta_{2}\right)$, we use a discretization angle of $\Delta \theta=\frac{\pi}{90}=2^{\circ}$ for the gradient extraction (eq. 13).

The first result presented in Fig. 8 shows the behavior of our anisotropic diffusion method with different $(k, h)$ parameters on stripes created by a brush on a painting. These results depict the coherence of our diffusion scheme because lineaments are a prolonged anisotropically diffusion scheme whereas structures are preserved and enhanced. Especially using $k=0.2$ and $h=0.1$ (Fig. 8(b) and (f)), the characters are perfectly visible and the cart remains intact. Concerning the other parameters (Fig. 8(c) and (d) with the close up in Fig. 8(g) and (h)), lineaments are diffused in the correct directions and we can remark that the method smooths more edges having small gradients using $k>0.2$ and $h>0.1$.

The second image shown in Fig. 9(b) is a natural image contaminated by a Gaussian noise $(\sigma=10)$. We aim to regularize this picture preserving edges as far as possible. As presented in Fig. 9(g), (h) and (i), comparing the absolute error between the original image and the regularized image, our algorithm preserves better edges than tensorial results ${ }^{10,11}$ (also the mean absolute error is smaller with our method). To obtain a better visualization, note that the absolute error images are corrected following a curve process on the image histogram, as presented in Fig. 9(c). This visualization process is the same for each absolute error image presented in this paper.

The third noisy image presented in Fig. 10(b) contains a random Gaussian noise of standard deviation $\sigma=20$. Due to this high noise and the texture, this image is particularly difficult to regularize correctly preserving the

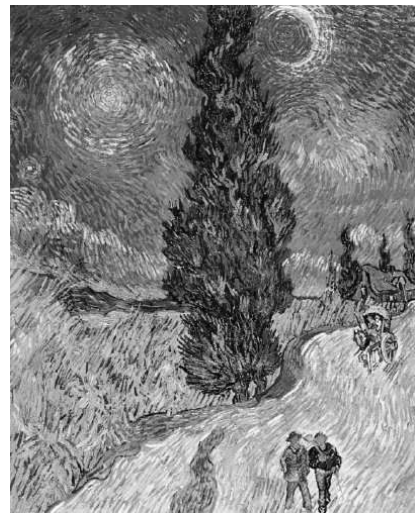

(a) "Road with Cypress and Star" by van Gogh $400 \times 503$

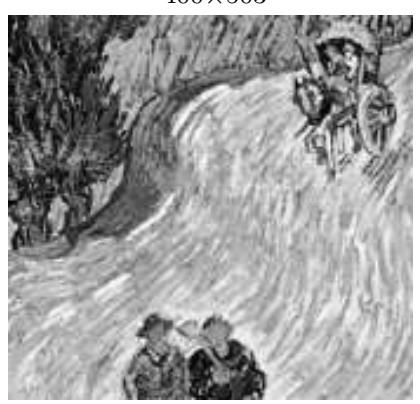

(e) Enlargement of (a)

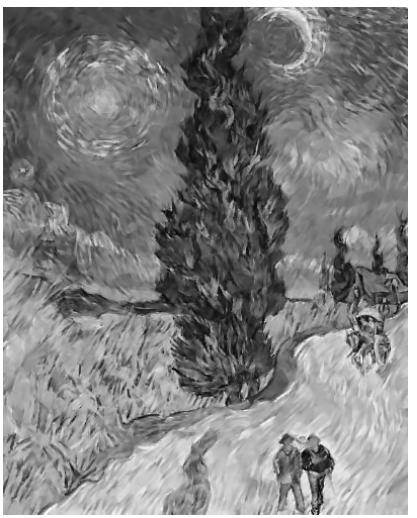

(b) $\mu=10, \lambda=1, \Delta \theta=\frac{\pi}{90}$ 50 iterations

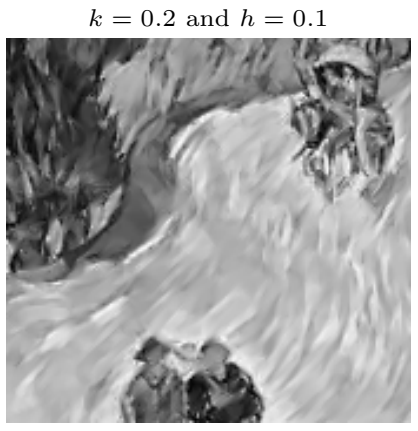

(f) Enlargement of (b)

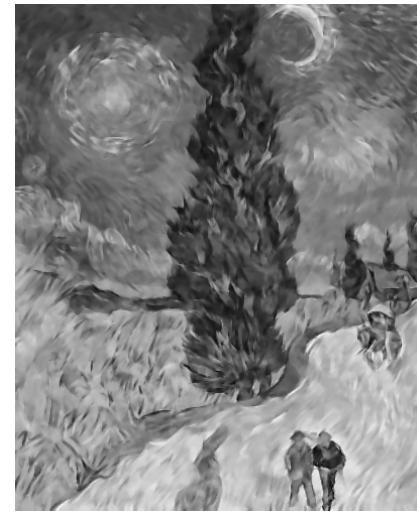

(c) $\mu=10, \lambda=1, \Delta \theta=\frac{\pi}{90}$ 50 iterations

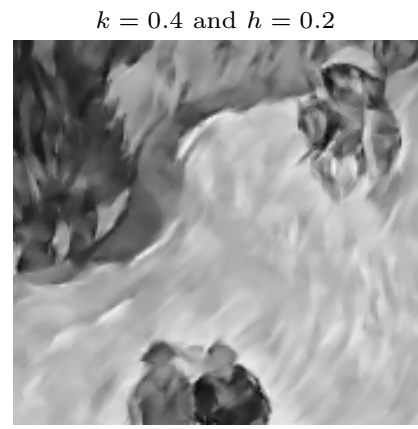

(g) Enlargement of (c)

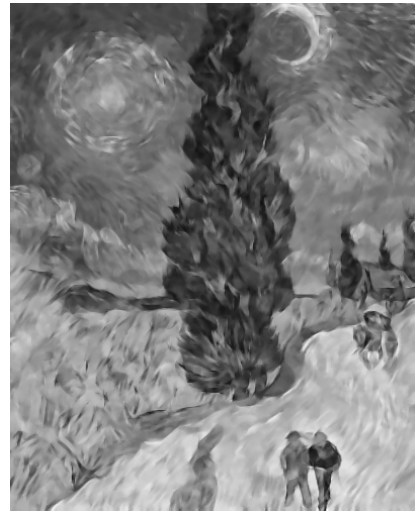

(d) $\mu=10, \lambda=1, \Delta \theta=\frac{\pi}{90}$ 50 iterations

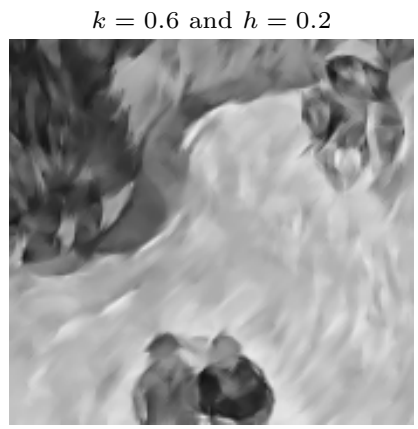

(h) Enlargement of (d)

Figure 8. Impact of the $(k, h)$ values on our diffusion scheme using same other parameters and same iterations number. 


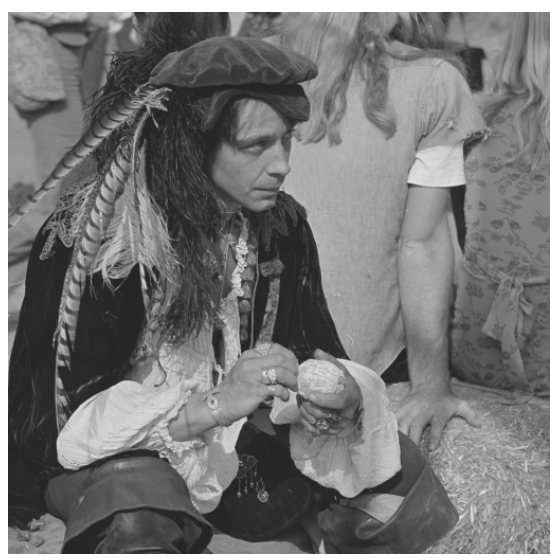

(a) Image of the Man, $512 \times 512$

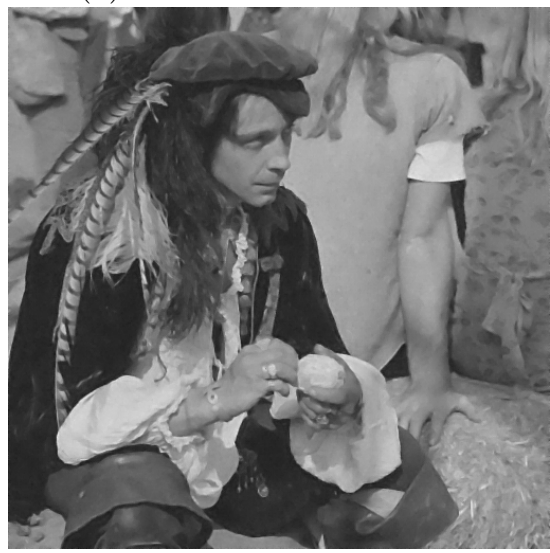

(d) Tensorial result ${ }^{10}$

$\sigma=1, \rho=0.7,20$ iterations

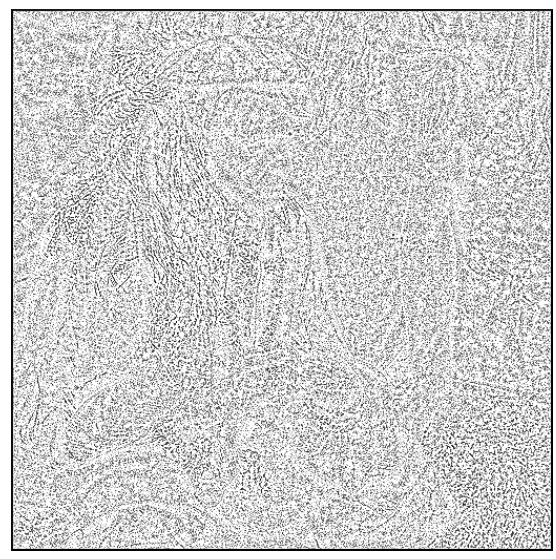

(g) Absolute error of (d)

Mean absolute error $=0.029$

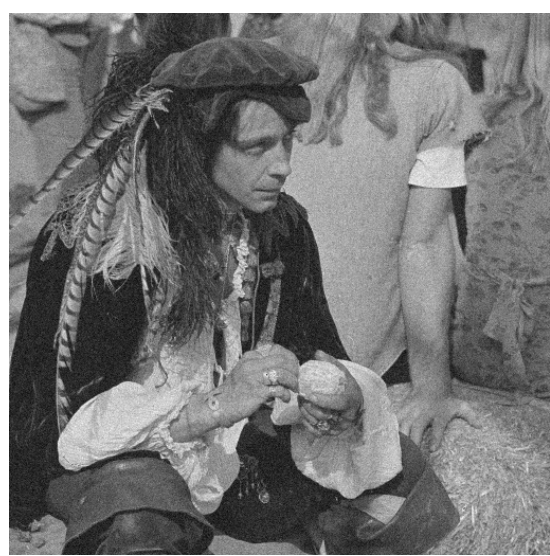

(b) Noisy image, $\sigma=10$

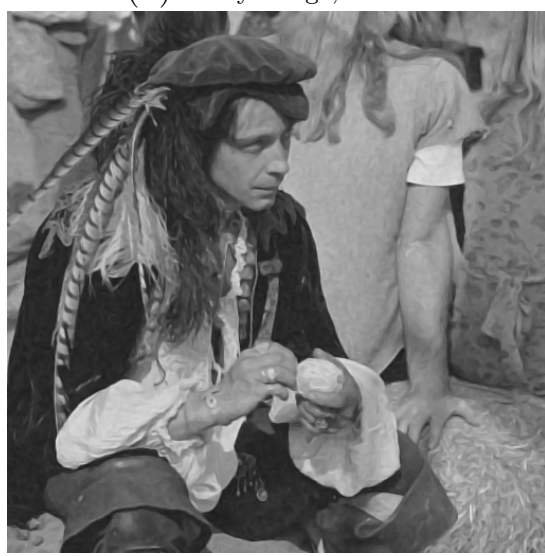

(e) Tensorial result ${ }^{11}$

$\sigma=1, \rho=0.7,20$ iterations

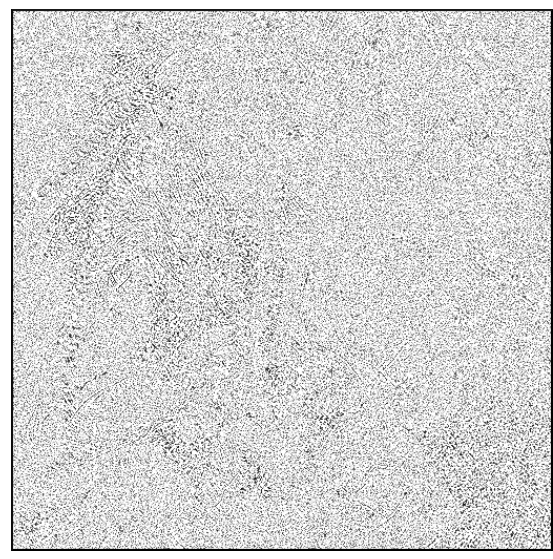

(h) Absolute error of (e)

Mean absolute error $=0.031$

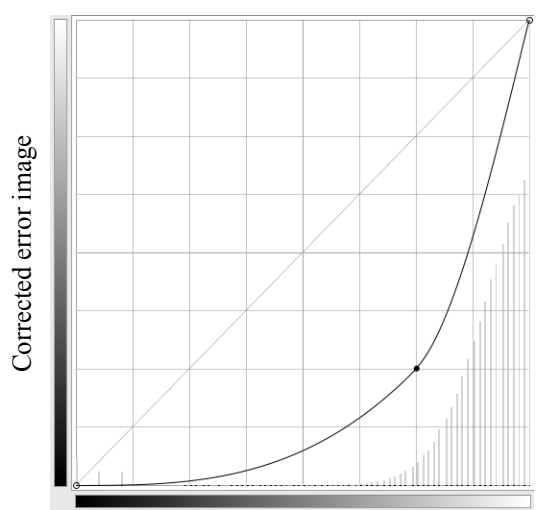

Original error image

(c) Correction curve of the error images

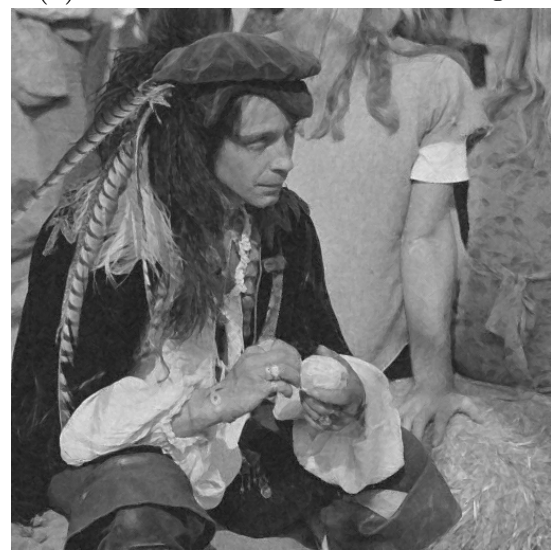

(f) Our result

$\mu=5, \lambda=1, \Delta \theta=\frac{\pi}{90}, 10$ iterations

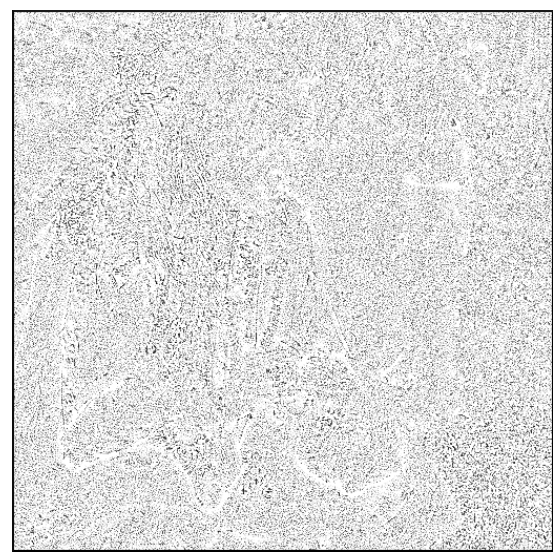

(i) Absolute error of (f)

Mean absolute error $=0.024$

Figure 9. Image regularization and absolute error. Absolute error are negative images.

thin texture. For each filter, we choose the parameters that gives the best results. In order to obtain comparative results, we choose the same larger (i.e. standard deviation) of the Gaussian for approaches using this function (i. e. $\sigma=\mu=1$ for $\left.^{7,9-11}\right)$. We compare our result with the $M C M^{8}$, the methods of Perona-Malik, ${ }^{5}$ Alvarez et al. ${ }^{7}$, tensorial driven diffusion ${ }^{9-11}$ and Magnier et al. ${ }^{21}$. Some results are presented in Fig. 11 with an enlargement. 


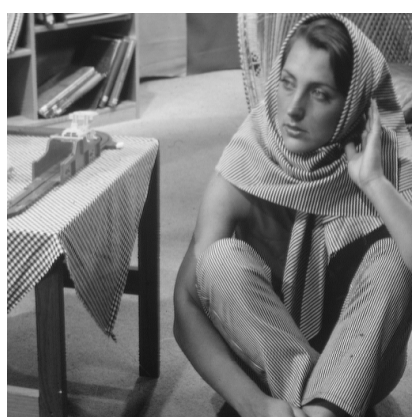

(a) Image of Barbara $512 \times 512$

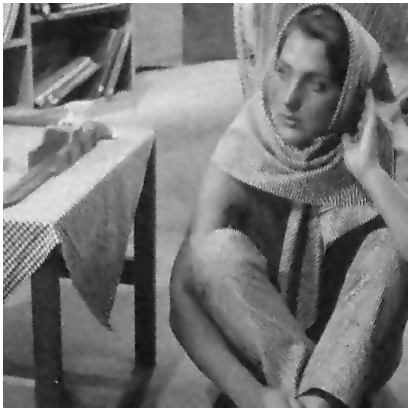

(e) Alvarez et al. diffusion ${ }^{7}$

$\sigma=1,20$ iterations, $K=0.02$

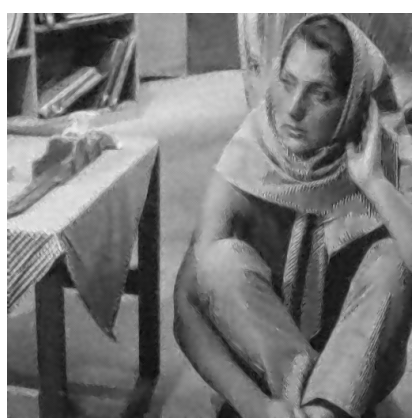

(i) Magnier and al. method ${ }^{21}$

$\mu=5, \lambda=1,10$ iterations

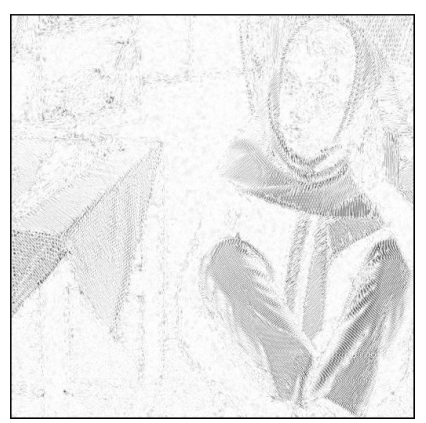

(m) Absolute error of (i)

Mean absolute error $=0.039$

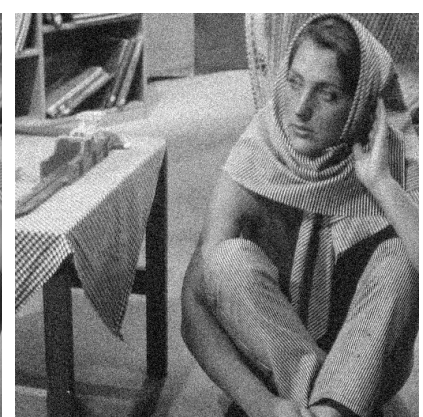

(b) Degraded image with a Gaussian noise: $\sigma=20$

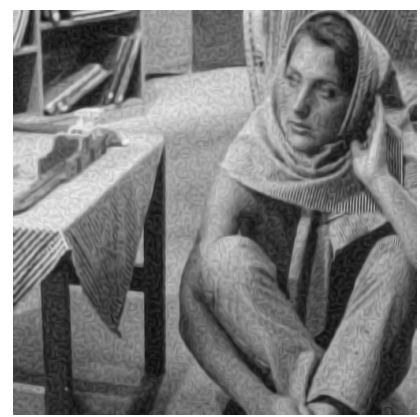

(f) Weickert's result ${ }^{9}$

$\sigma=1, \rho=0.7,50$ iterations

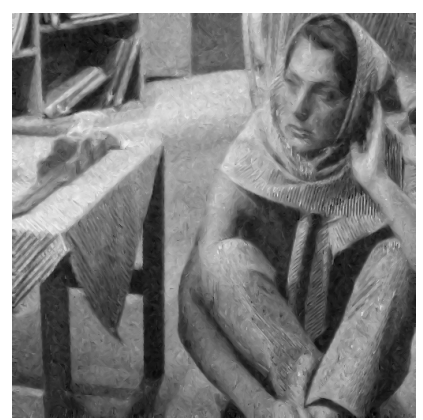

(j) Eq. 16, $K_{\{1,2\}}=0.6$,

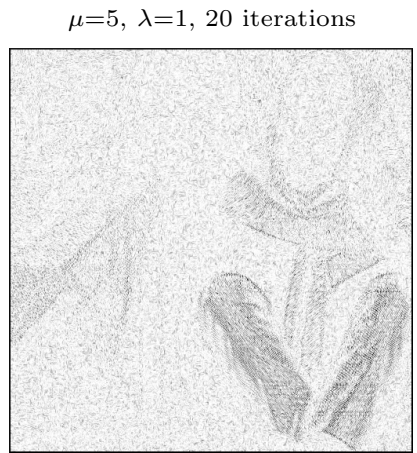

(n) Absolute error of (h)

Mean absolute error $=0.034$

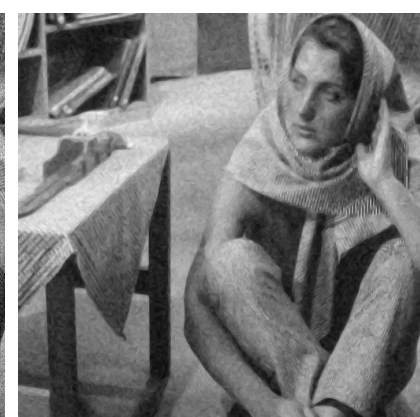

(c) $M C M$ diffusion scheme ${ }^{8}$ 20 iterations

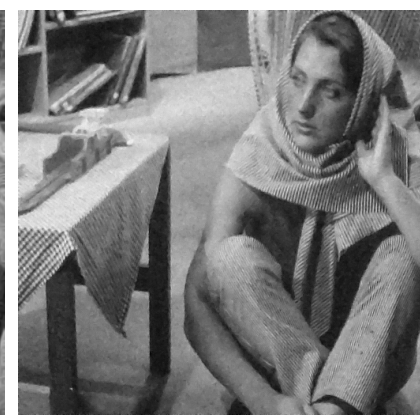

(g) Tensorial result ${ }^{10}$

$\sigma=1, \rho=0.7,20$ iterations

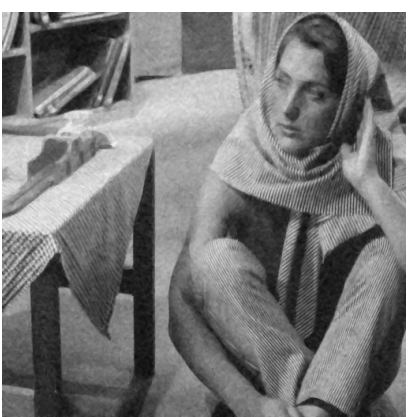

(k) Sub result, $k=0.6, h=0.2$,

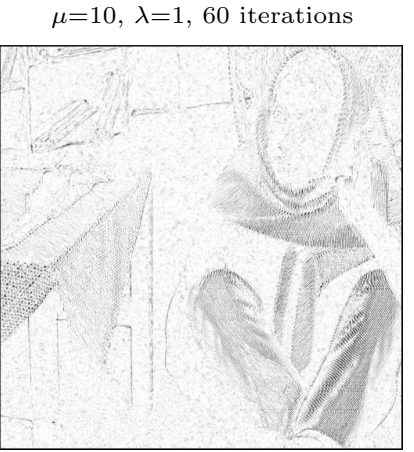

(o) Absolute error of (k)

Mean absolute error $=0.037$

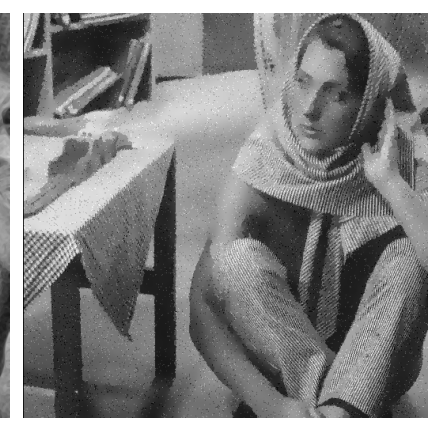

(d) Perona-Malik diffusion ${ }^{5}$ $K=0.05,100$ iterations

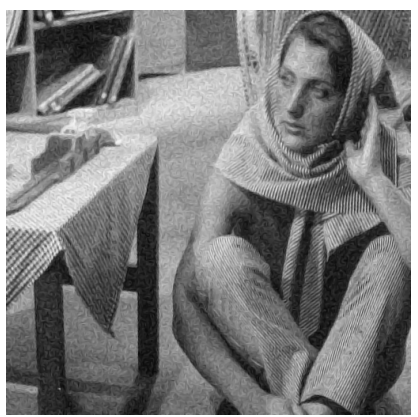

(h) Tensorial result ${ }^{11}$

$\sigma=1, \rho=0.7,20$ iterations

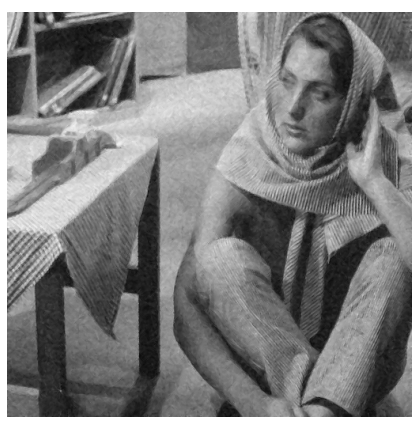

(1) Our result, $k=0.6, h=0.2$, $\mu=5, \lambda=1,15$ iterations

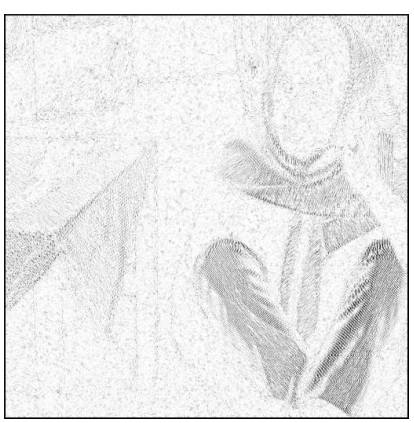

(p) Absolute error of (l)

Mean absolute error $=0.034$

Figure 10. Enhancement of Barbara image by different PDE methods. 


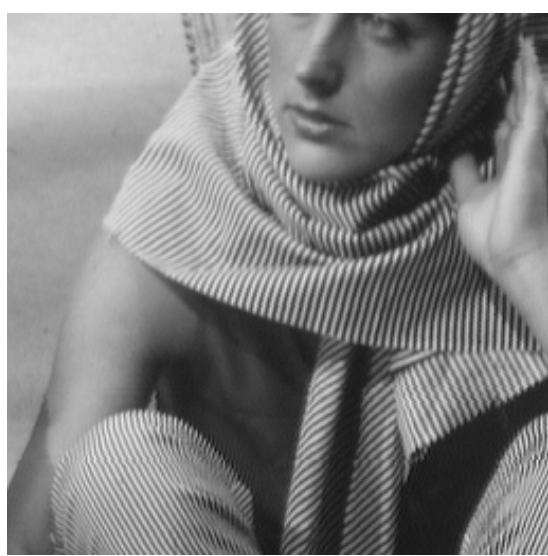

(a) Barbara, original

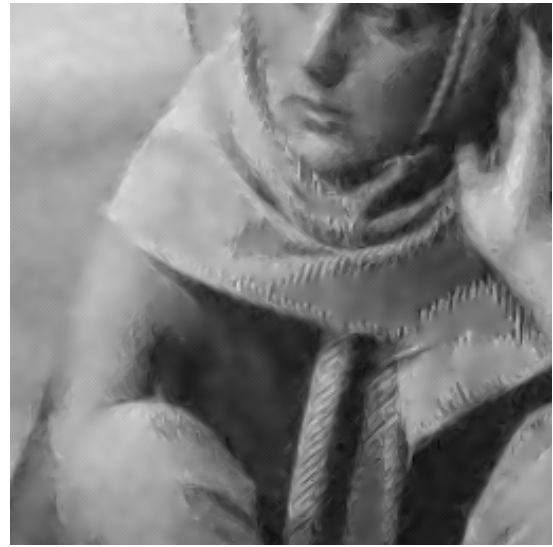

(d) Anisotropic diffusion in edges region ${ }^{21}$

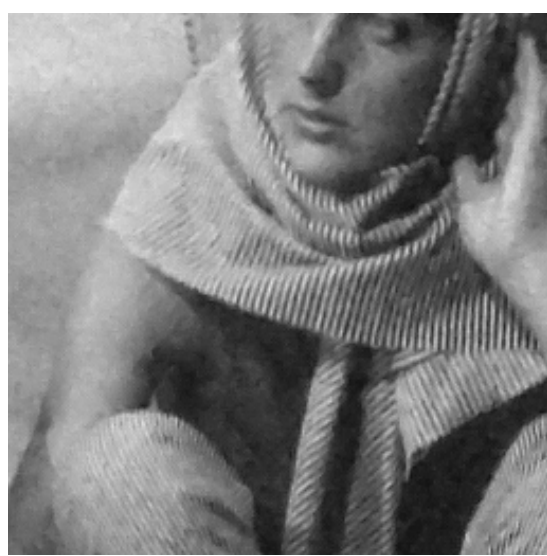

(b) Tensorial result ${ }^{10}$

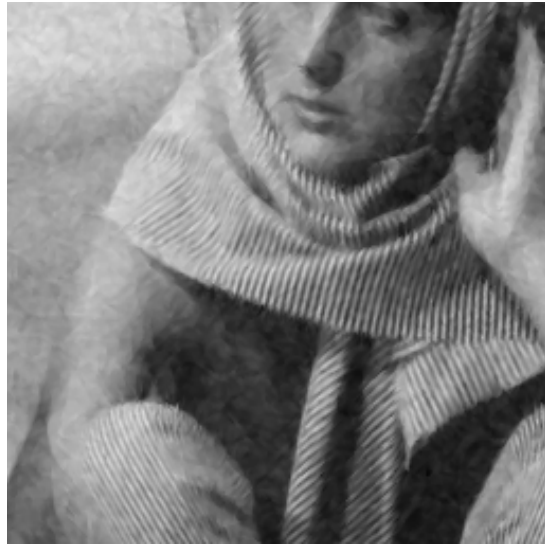

(e) Sub pixel resolution

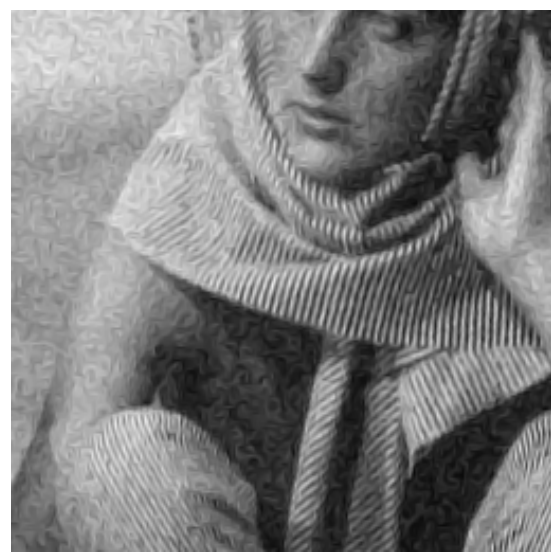

(c) Tensorial result ${ }^{11}$

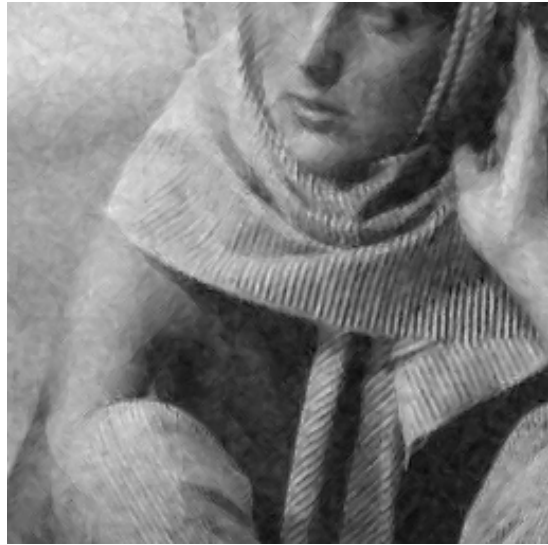

(f) Our result

Figure 11. Enlargement of results presented in Fig. 10.

It is easy to remark that $M C M$ and Perona-Malik models do not remove correctly the noise. Algorithm of Alvarez et al. loses the texture and creates artifacts at position of edges. Tensors ${ }^{9,11}$ diffusion creates a fiber effect in homogeneous regions due to the high noise (see details in Fig. 11(c)). Tensorial result of Tschumperlé ${ }^{10}$ gives a good result even if this method is known to distort corners. As the method of Magnier et al. ${ }^{21}$ diffuses anisotropically only at level of edges, the diffusion results presented in Fig. 10(i) and Fig. 11 show a good
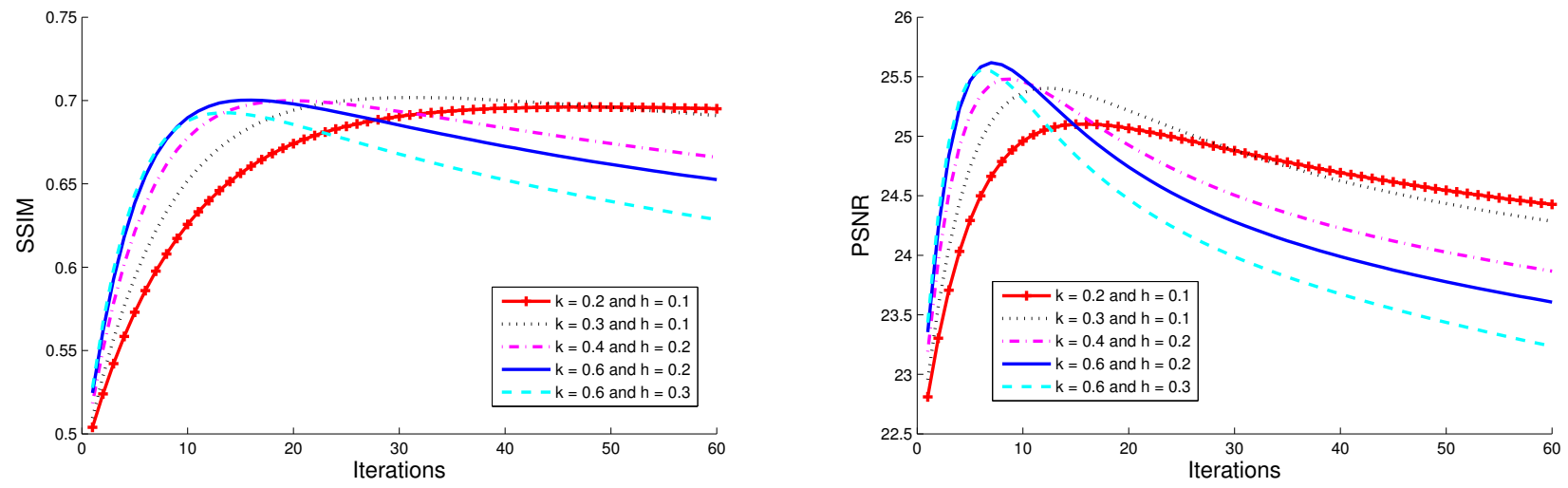

Figure 12. Mesures of the results in function of the evolution of the parameters $(k, h)$ and the number of iterations. 


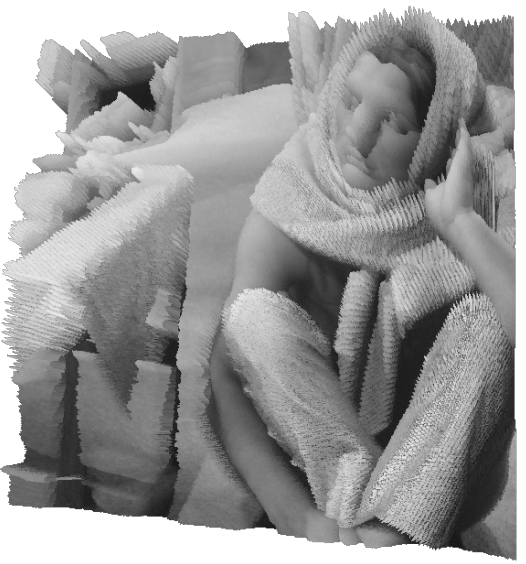

(a) Barbara, original

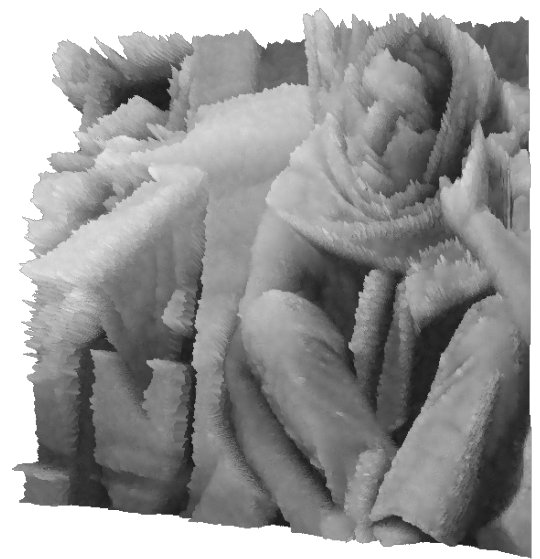

(d) Anisotropic diffusion in edges region ${ }^{21}$

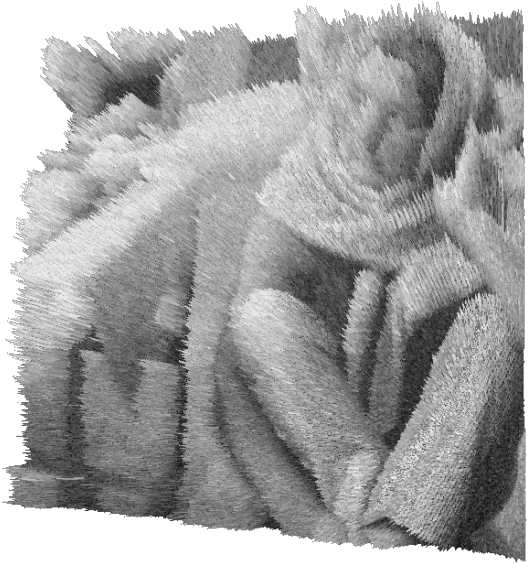

(b) Noisy image

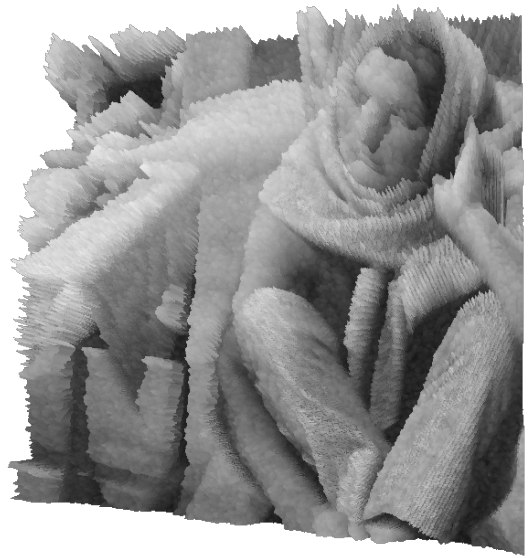

(e) Sub pixel resolution

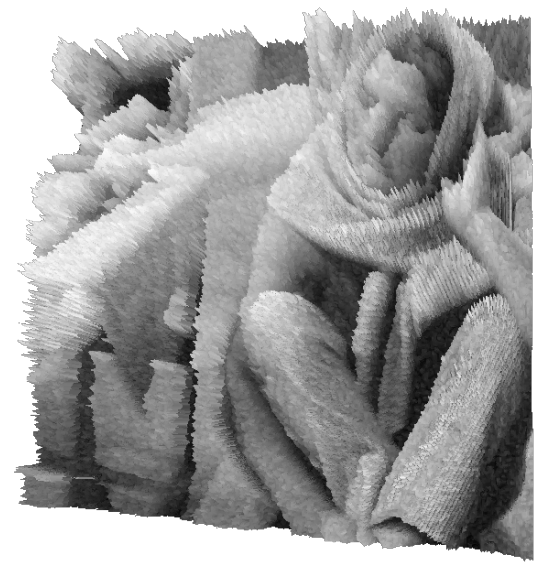

(c) Tensorial result ${ }^{11}$

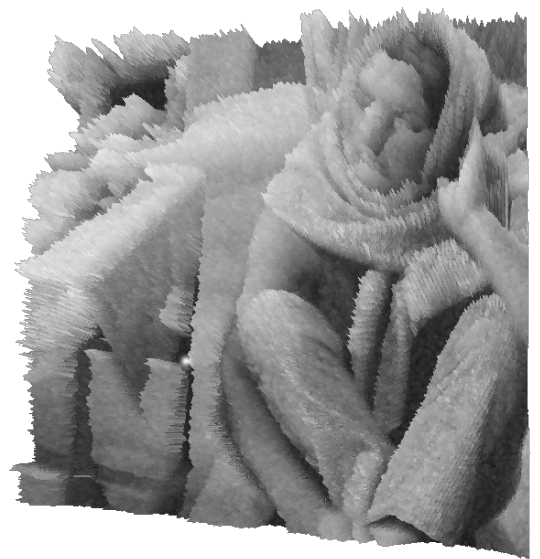

(f) Our result

Figure 13. Image surface of different results presented in Fig. 10.

regularization inside homogeneous regions and a loss of textures. Diffusing everywhere only in the two direction $\left(\theta_{1}, \theta_{2}\right)$, this method does not enable to preserve neither texture, nor small objects (Fig. 10(j)). Our algorithm preserves correctly edges (Fig. 10(l)), does not create undesirable fiber effect inside homogeneous regions and enables a regularization of stripes textures with of the use of thin half kernels.

Fig. 10(m), (n), (o) and (p) depicts the absolute error of different methods (i.e. the absolute error between the corrected image and original image of Barbara Fig. 10(a)). The tensorial method of ${ }^{11}$ brings a lot of errors especially inside homogeneous regions whereas Magnier et al. ${ }^{21}$ approach is able to keep correctly edges but removing the texture (respectively Fig. 10(h) and (i)). Fig. 10(m) shows that our algorithm preserves most of edges and small objects (book, tool on the table, face...) while it does not lose all the texture (on the head, the bust...). Also, Fig. 13 provides another point of view for the results evaluation and the preservation of stripe textures with the image surface. Thus, homogeneous regions, sharped edges and stripe textures are visually enhanced. Indeed, we can see that our result is more regular in homogeneous regions than tensorial approach ${ }^{11}$ and that edges are sharped. Moreover, this visualization enables us to ascertain that the arm and the face of Barbara are correctly smoothed (on the nose and the cheeks). Finally, at several locations, the texture is correctly preserved, contrary to the method of Magnier et al. ${ }^{21}$.

In another experiment shown in Fig. 10(k), our diffusion scheme is applied to a sub-pixel image ${ }^{25}$ (i.e. the image is resized from $512 \times 512$ to $1024 \times 1024$ using interpolation) and thus allowing the use of thiner half kernels: $\mu=10$ and $\lambda=1$ (instead of $\mu=5$ ). This practice enables to better regularize stripe textures (see enlargements in Fig. 11(e) and 3D elevation in Fig. 13(e)). Even if some errors presented in Fig. 10(o) are caused by the 


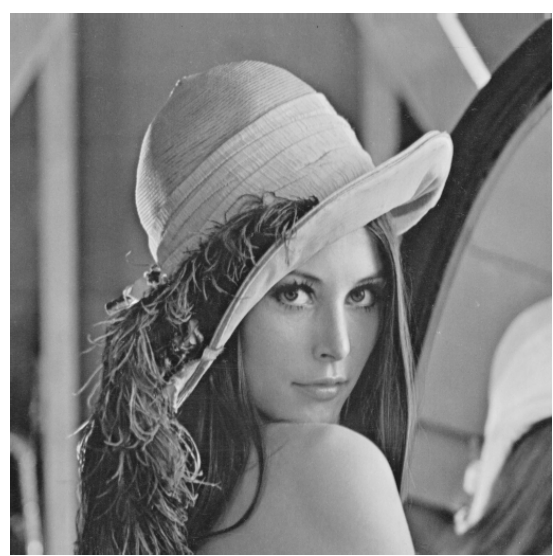

(a) Image of Lena $512 \times 512$

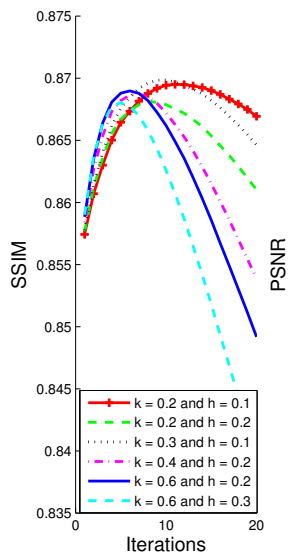

(d) SSIM and PSNR

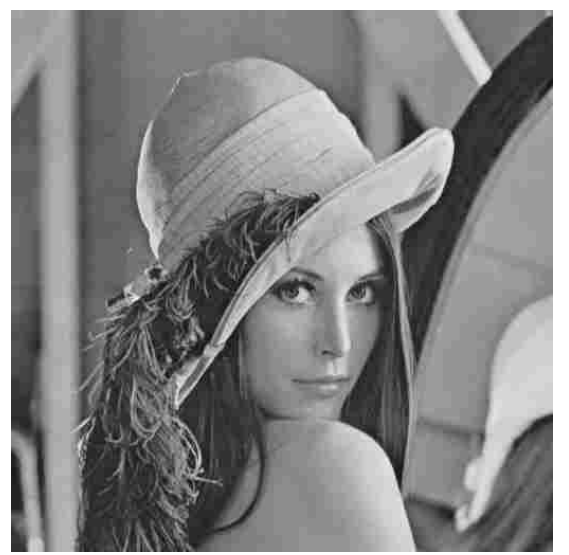

(b) JPEG compression

JPEG quality factor $=15$

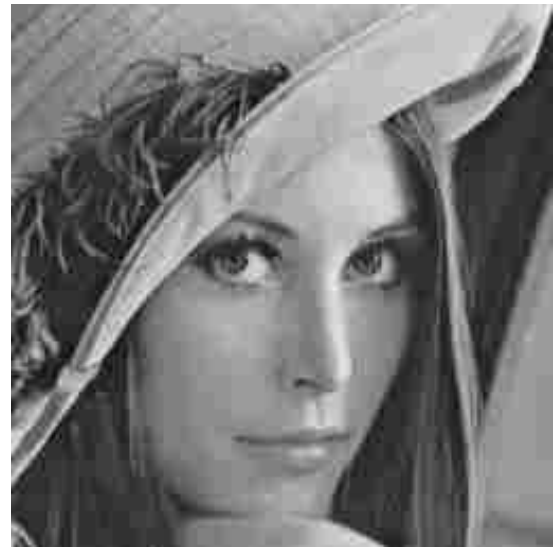

(e) Enlargement of (b)

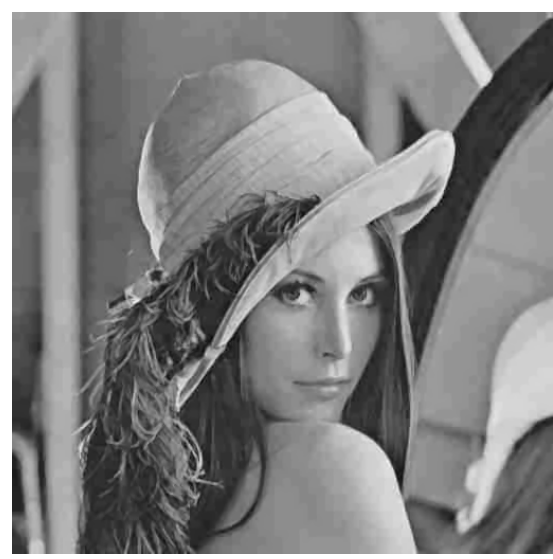

(c) $\mu=5, \lambda=1, \Delta \theta=\frac{\pi}{90}$

10 iterations, $k=0.3$ and $h=0.1$

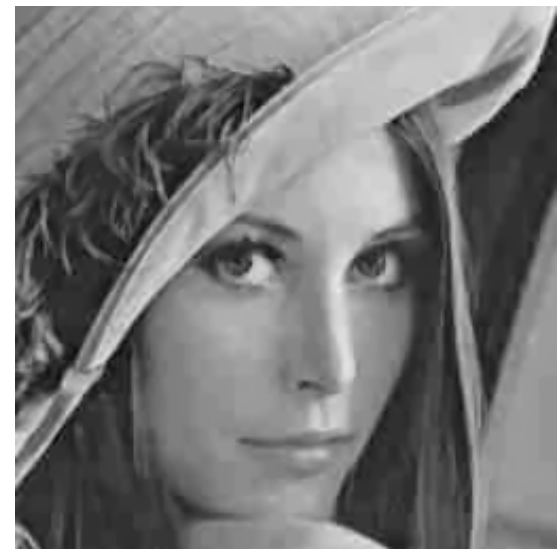

(f) Enlargement of (c)

Figure 14. Suppression of JPEG compression artifacts.

resizing back of the regularized image (i.e. the decimation coming back to the original image size of $512 \times 512$ ), the thin stripes are better regularized than with other methods.

Image of Lena presented in Fig. 14 is concerned with significantly of compression artifacts and block splitting effect. The JPEG quality factor is equal to 15, which degrades the image. Block effects inherent to the compression are visible, especially in the enlargement presented in Fig. 14(b). After 10 iterations using our diffusion method, the image is enhanced, and small details are preserved (see Fig. 14(c)). Fig. 14(d) shows which parameters $(k, h)$ are better adapted in term of quality measures for this type of image restoration, although these measures do not directly always the visual quality.

We have tested different values of the couple $(k, h)$ and when $k<h$, the diffusion result in bluring edges. When $k \leqslant 0.2$, the diffusion process preserves well edges but creates also irregular artifacts due to the control functions which do not smooth sufficiently the noise. Generally, the choice of our half kernel filters parameters $(\mu, \lambda)$ is done in function of the noise level. In order to preserve small objects, we can choose for the length of our filter $\mu=5$. However, the width which corresponds to the derivation filter depends of the noise level. Regularly, we choose $\lambda=1$, it enables to keep precisely edges but if the noise is higher, we can choose a larger parameter. For example, when lighting conditions are not optimal as the next image presented in Fig. 15 leads to a natural image containing high noise.

Indeed, the last image shown in Fig. 15(a) illustrates that our algorithm is able to regularize images containing important noise while preserving different structures (Fig. 15(d)). The enlargement in Fig. 15(e) shows a corner 


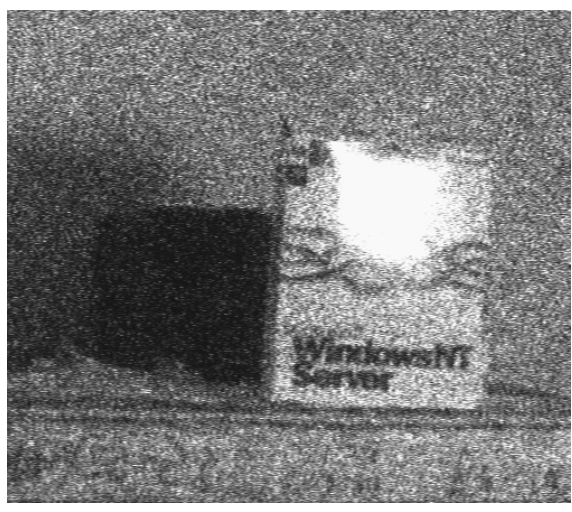

(a) Real noisy image $508 \times 440$

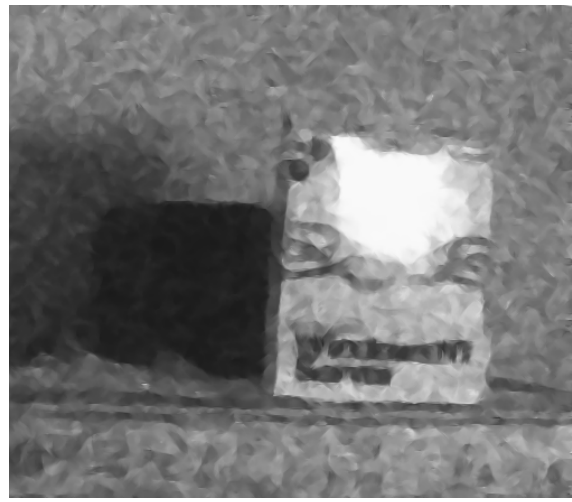

(d) $\mu=5, \lambda=2, \Delta \theta=\frac{\pi}{90}, 100$ iterations

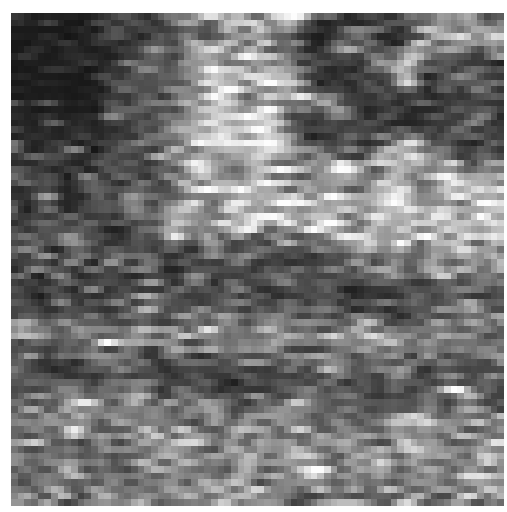

(b) Enlargement in (a)

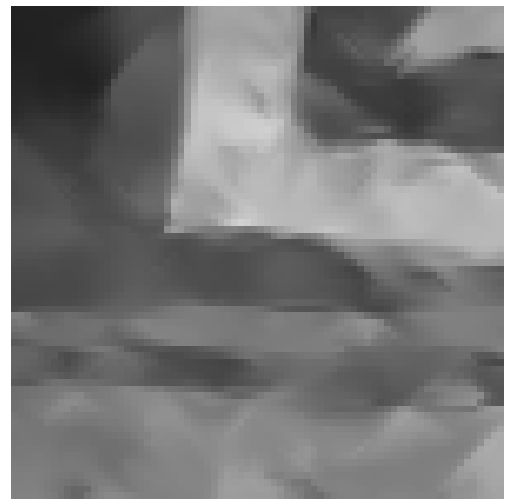

(e) Enlargement of (d)

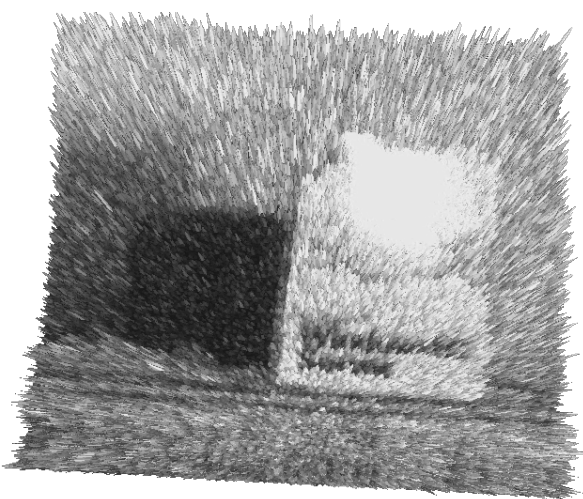

(c) 3D elevation of (a)

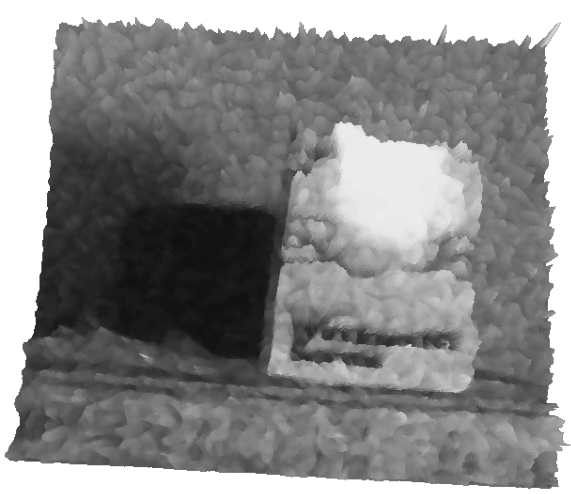

(f) 3D elevation of (f)

Figure 15. Real image regularization. The enlargements of the images represent the corner on the bellow left of the box.

well regularized, even after 100 iterations, whereas it is visible with difficulty in the original image (see Fig. 15(b)). Finally, the image surface shows that regularized edges are sharped compared to the original image (Fig. $15(\mathrm{c})$ and (f)).

\section{CONCLUSION AND FUTURE WORKS}

In this paper, we have presented a new image regularization method, based on half anisotropic Gaussian kernels. To make it more efficient, we have introduced new control functions which enables a diffusion process preserving both edges and corners in the image. The main advantages of our method is that it is based on half kernels, so it extracts two direction of edge diffusions which allows a preservation of small objects. Moreover, diffusing also in a third direction, corresponding to the bisector of the edges orientations, well controlled, avoid an irregular smoothing in homogeneous regions and undesired artifacts. Promising experimental results have been achieved and shown in this paper. Comparing the absolute error with other PDE method, they open new perspectives in PDE-based image processing as image inpainting and an extension to color images.

Future works will include automatic diffusion/detection parameters estimation, that would lead to an unsupervised restoration algorithm based on a multi-scale anisotropic half kernel. The execution times using non-optimized $\mathrm{C} / \mathrm{C}++$ code was about $42 \mathrm{~s}$ per image of $512 \times 512$ (i.e. 23 s for the gradient estimation with a discretization angle of $\frac{\pi}{90}=2^{\circ}$ and $19 \mathrm{~s}$ concerning the computation of the diffusion directions and the diffusion itself with 50 iterations) with 1 processor running at $2.4 \mathrm{GHz}$ and 4 Go of RAM. Therefore, the method code could be enhanced, in particular for the rotation of the images using a recursive image rotation ${ }^{26}$ instead of a $^{2}$ simple interpolation ${ }^{18}$. 


\section{REFERENCES}

[1] Weickert, J., "Anisotropic diffusion in image processing.," Teubner-Verlag, Stuttgart, Germany (1998).

[2] Aubert, G. and Kornprobst, P., [Mathematical problems in image processing: partial differential equations and the calculus of variations (second edition)], vol. 147, Springer-Verlag (2006).

[3] Caselles, V. and Morel, J., "Introduction to the special issue on partial differential equations and geometrydriven diffusion in image processing and analysis.," IEEE TIP 7(3), 269-273 (1998).

[4] Koenderink, J., "The structure of images," Biological cybernetics 50(5), 363-370 (1984).

[5] Perona, P. and Malik, J., "Scale-space and edge detection using anisotropic diffusion," IEEE TPAMI 12, 629-639 (1990).

[6] Kornprobst, P., Deriche, R., and Aubert, G., "Nonlinear operators in image restoration," in [ICVPR], 325-331 (1997).

[7] Alvarez, L., Lions, P.-L., and Morel, J.-M., "Image selective smoothing and edge detection by nonlinear diffusion, ii," SIAM J. of Num. Anal. 29(3), 845-866 (1992).

[8] Catté, F., Lions, P., Morel, J., and Coll, T., "Image selective smoothing and edge detection by nonlinear diffusion," SIAM J. of Num. Anal. , 182-193 (1992).

[9] Weickert, J., "Coherence-enhancing diffusion filtering," IJCV 31(2), 111-127 (1999).

[10] Tschumperlé, D. and Deriche, R., "Vector-valued image regularization with pdes: A common framework for different applications," IEEE TPAMI , 506-517 (2005).

[11] Tschumperlé, D., "Fast anisotropic smoothing of multi-valued images using curvature-preserving PDE's," IJCV 68(1), 65-82 (2006).

[12] Freeman, W. T. and Adelson, E. H., "The design and use of steerable filters," IEEE TPAMI 13, 891-906 (1991).

[13] Jacob, M. and Unser, M., "Design of steerable filters for feature detection using canny-like criteria," IEEE TPAMI 26(8), 1007-1019 (2004).

[14] Perona, P., "Steerable-scalable kernels for edge detection and junction analysis," IMAVIS 10(10), 663-672 (1992).

[15] Sha'ashua, A. and Ullman, S., "Structural Saliency: The Detection of Globally Salient Structures Using Locally Connected Network," in [ICCV], 321-327 (1988).

[16] Simoncelli, E. and Farid, H., "Steerable wedge filters for local orientation analysis," IEEE TIP 5(9), 1377$1382(1996)$.

[17] Canny, F., "A computational approach to edge detection," IEEE TPAMI 8(6), 679-698 (1986).

[18] Montesinos, P. and Magnier, B., "A New Perceptual Edge Detector in Color Images," in [ACIVS], 2, 209-220 (2010).

[19] Deriche, R., "Recursively implementing the gaussian and its derivatives," in [ICIP], 263-267 (1992).

[20] Palomares, J. L., Montesinos, P., and Diep, D., "A New Affine Invariant Method for Image Matching," in [IEEE SPIE (3DIP) and Applications], (2012).

[21] Magnier, B., Montesinos, P., and Diep, D., "A new region-based pde for perceptual image restoration," in [VISAPP], 56-65 (2012).

[22] Magnier, B., Montesinos, P., and Diep, D., "Fast Anisotropic Edge Detection Using Gamma Correction in Color Images," in [IEEE 7th ISPA], 212-217 (2011).

[23] Magnier, B., Montesinos, P., and Diep, D., "Texture Removal by Pixel Classification using a Rotating Filter," in [IEEE 36th ICASSP], 1097-1100 (2011).

[24] Wang, Z., Bovik, A., Sheikh, H., and Simoncelli, E., "Image quality assessment: From error visibility to structural similarity," IEEE TIP 13(4), 600-612 (2004).

[25] Montesinos, P. and Dattenny, S., "Sub-pixel accuracy using recursive filtering," in [SCIA], 523-530 (1997).

[26] Condat, L. and Van De Ville, D., "Fully reversible image rotation by 1-d filtering," in [ICIP], 913-916 (2008). 